\title{
Coverage Probability using EM waves for UWSN in Shadowing Environment
}

\author{
Anvesha Katti \\ Jawaharlal Nehru University \\ New Delhi \\ India
}

\author{
D. K. Lobiyal \\ Jawaharlal Nehru University \\ New Delhi \\ India
}

\begin{abstract}
A channel model is introduced which considers shadowing fading on coverage probability for underwater sensor networks using electromagnetic signals. A model for finding the probability of detection and coverage using log normal shadowing fading has been derived. A comparative study between the sensing channel model and existing model for terrestrial sensor networks is also presented. It is observed the introduced sensing channel model uses far less number of sensors for coverage of underwater networks in comparison to terrestrial networks.
\end{abstract}

\section{General Terms}

\section{Sensor Networks}

\section{Keywords}

Coverage probability, detection probability, shadowing, fading, underwater sensor networks

\section{INTRODUCTION}

A wireless sensor network (WSN) consists of self governing sensor nodes to oversee physical or environmental conditions such as temperature, sound, etc [9]. An Underwater Sensor Network (UWSN) is a WSN under water consisting of sensor nodes and Autonomous Underwater Vehicles (AUVs) equipped with sensors which are spread across and pass data through multiple hops to a main location called sink. UWSN can self-organize and adapt themselves to the ocean environment. They perform synergic monitoring and analysis of the ocean [1].

Coverage is one of the many demanding areas in a WSN. Coverage in a broad sense refers to how well sensors can record the events in a field. It is also a measure of Quality of Service (QoS) in a sensor network. The coverage requirements are application and environment dependent and can differ accordingly [2, 3]. Different applications may require different degrees of sensing coverage; for example, some applications may require only one sensor that monitors a location, while other applications may require higher number of sensors for the same $[4,5]$.

Sensor nodes are normally taken to be static in most of the work studying coverage, i.e. they stay in the same position once they are deployed. In this work, sensor nodes are considered to be randomly deployed and static. It is important to know apriori the minimum number of nodes that provide a fair level of coverage. Myriad applications involving sensor networks require acoustic signals, seismic signals, radio waves, light waves, or magnetic field in the sensing area to become aware of an event or an object [6]. Environmental factors like noise and interference affect these signals. These signals have added power loss due to the mentioned factors besides path loss. This added power loss results in large disparity in the received signal strength. The disparity in the received signal strength due to hindrance in propagation path is known as shadowing which negatively affects the quality of sensing coverage $[13,21]$.

One of the demanding issues of UWSN is efficient underwater communication among the nodes. Underwater communication systems can use acoustic, optical or electromagnetic (EM) waves to transmit information but each of these signals have their own advantages and disadvantages. A major limiting factor for efficient communication underwater using acoustic waves is the slow speed in water i.e. about $1500 \mathrm{~m} / \mathrm{s}$ although the attenuation is very low. EM waves on the other hand despite having a conducting nature and very high attenuation, have much faster propagation speed which is a boon for rapid and dynamic communication among the nodes. They also have the distinct advantage of reducing latency. Above a frequency of $10 \mathrm{KHz}$ EM waves can propagate even hundred times faster than acoustic waves which can prove to be much more advantageous for command latency and network protocols. Besides this, the EM waves have the ability to be environment friendly communication system of the future as also they are one of the most practical choices for cross boundary air-water underwater communication. On the downside, EM waves require high transmission power and large antennae as they are affected by high attenuation in water at high frequency. Although the acoustic waves have very less attenuation they has many constraints which include time-varying multi-path propagation, limited and distance-dependent bandwidth, and low speed which result in a poor quality and high latency communication channel [20]. Different materials cause different amount of delays due to attenuation. Very low frequencies (10 to $30 \mathrm{kHz}$ ) need to be used for communication in sea water as the attenuation of EM waves is very high in the order of 3.5 to $5 \mathrm{~dB}$ per metre [7]. Suspended particles as also with reflection and refraction have less interference with EM waves [16, 17]. Good communication between the nodes is dependent for a large part on the coverage of the network area by the sensor nodes. A sensor can keep a track of all targets that are in its sensing range. Therefore the better the coverage the better the data reception by all the sensors that fall in its communication range.

Most of the literature until now has considered coverage problem for terrestrial sensor networks. The authors believe no study has yet focused on shadowing effects on network coverage in UWSN using EM waves.

A new sensing channel model has been proposed which considers shadowing effects for UWSN using electromagnetic waves. Coverage probability in the presence of shadowing is derived based on the received signal strength using a mathematical model. A comparative study between the proposed sensing channel model and existing sensing models, 
namely Elfes sensing model [10] in terms of network coverage has been carried out [21].

The rest of the paper is organised as follows. Section 2 gives the research work related to coverage problem detailed in literature. Section 3, proposes the sensing channel model. Section 4 presents analytical and simulation results for the proposed model. The conclusion of the work is given in Section 5.

\section{RELATED WORK}

Although research in the area of sensing coverage for UWSN has received almost no attention, a lot of work is reported for terrestrial sensor networks. Tsai et al. [6] have investigated sensing coverage under shadowing-fading environment with asymmetric sensing ability of sensors in a randomly deployed wireless sensor network. Hunt et al. [17] describe work relating to EM waves through soil and water where underground communication characteristics of wireless underground sensor networks are studied. Elrashidi et al. [7] compares path loss due to attenuation of electromagnetic waves in sea with path loss due to attenuation of electromagnetic wave in pure water. Weldon et al. [9] studies the proliferation of electromagnetic waves through soil of frequency from 1 to $2 \mathrm{GHz}$. K. Hunt at el. [15] review the characteristics of radio waves underwater and between water and air interface. They also shed light upon noise due to transmission, reflection from the interface surface between air, water and signal attenuation. In case of terrestrial sensor networks most of the models focus on coverage algorithms and issues of estimating number of sensors to be set up but do not take environmental conditions into consideration.

\section{CHANNEL MODEL}

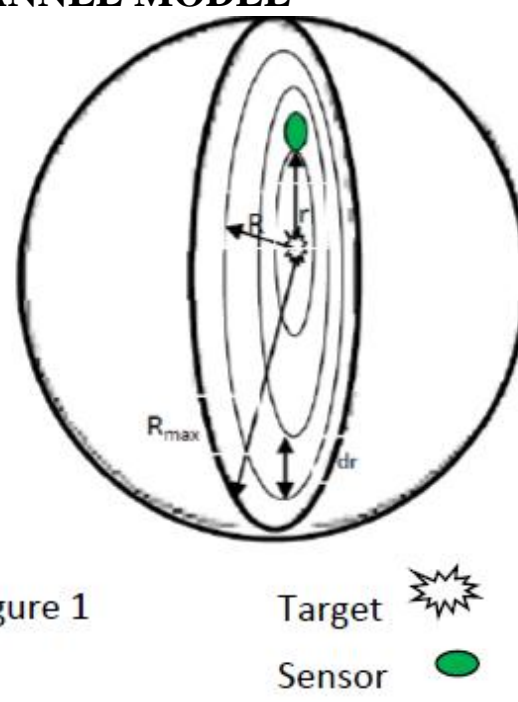

As shown in the figure above, a sensor is located at distance rfrom the target located at the origin of the circle. Rmax is the maximum sensing range of a sensor. Ris an average sensing radius of the sensor.

$N$ uniformly deployed sensors are assumed to be in a cubic sensing field of volume V. All sensors are homogenous with the same sensing threshold power $\lambda$ (in $\mathrm{dB}$ ). The sensing threshold is defined as the minimum strength required by the received signal that can be correctly decoded at the sensor. The transmit power, sensing threshold power, and power attenuation along propagation path determines the sensing range of a sensor.
The sensing signal power generated by an event is assumed to be $\rho_{\mathrm{t}}$ (in $\mathrm{dB}$ ). Log-normal shadowing fading has been adopted to construct the proposed sensing channel model. In the sensing model, it is assumed that each sensor has a constant sensing range $r$. The sensing region of a sensor is a sphere of volume $v=(4 / 3) \pi r^{3}$. A sensor can only sense and detect the events within the sensing range of its environment. A target is said to be covered if it is within the sensing volume of a sensor. (Refer to Figure 1.) The probability of target detection by an arbitrary sensor is defined as the ratio of sensing volume to network volume. Therefore, it can be expressed as $P_{\mathrm{d}}=\mathrm{v} / \mathrm{V}$, where $\mathrm{V}$ is network volume in which $N$ sensor nodes are deployed uniformly [21]. The probability $\left(P_{\mathrm{c}}\right)$ of target detection (Coverage Probability) by at least one of the $N$ sensors according to the disk sensing model can be expressed as

$P_{\mathrm{c}}=1-\left(1-P_{d}\right)^{\mathrm{N}}$

Since $[1-x]^{\mathrm{n}} \approx e^{-} n x$ for large $n$, the above equation can be rewritten as

$P_{c}=1-\exp \left(\frac{-N 4 \pi r^{3}}{3 V}\right)$

The profile of the received signal can be obtained from the transmitted signal using the channel model. Assumptions include a non uniform sensing range with $\mathrm{N}$ sensors uniformly arranged in a sphere with sensing field of volume $\mathrm{V}$ to develop a sensing channel model. All sensors are regarded homogenous having the equivalent sensing threshold power $\lambda$ (in $\mathrm{dB}$ ). The transmit power of a sensing signal, sensing threshold power, and power attenuation along the propagation path underwater determines the sensing range of the sensor [21]. The sensing signal power (transmitted power) generated by an event is assumed to be $\rho_{\mathrm{t}}($ in $\mathrm{dB})$.

According to log-normal shadowing the received signal power (d) (in $\mathrm{dB}$ ) at a sensor can be conveyed as in [11]:

$$
\begin{aligned}
& \rho_{L}(d)=\rho_{a}(d)+X_{\sigma} \\
& \rho_{a}(d)=\rho_{t}-\rho\left(d_{0}\right)-10 \eta \log _{10}\left(\frac{d}{d_{0}}\right)
\end{aligned}
$$

where $\eta$ is a path loss exponent that represents the rate at which the path loss increases with distance. $\rho\left(d_{0}\right)$ is the mean path loss at reference distance $d_{0}$ and $d$ is the distance between a sensor and its target. $X_{\sigma}$ is a Gaussian random variable (in $\mathrm{dB}$ ) with zero mean and variance $\sigma$ representing $\log$ normal shadowing effects in the propagation path. The received signal power $\rho_{\mathrm{L}}(d)$ is taken as a Gaussian distribution with an area mean power $\rho(d)$ (in $\mathrm{dB})[11]$.

\subsection{Coverage Using Log Normal Shadowing Fading}

The derivation for detection probability and coverage probability for EM signals using log-normal shadowing fading model to construct the proposed sensing channel model is presented. It is assumed that received signal power can vary in all the directions and a channel may be subject to log normal shadowing fading. This implies that the sensing radius of a sensor is not uniform in all directions [21]. Taking this into consideration and according to (3), (4) the received signal power $P_{\text {rec }}(r)($ in $\mathrm{dB}$ ) of a sensor at a distance $r$ from the target can be expressed as:

$P_{r e c}(\mathrm{dBm})=P_{t}(\mathrm{dBm})+G_{t}(\mathrm{~dB})+G_{r}(\mathrm{~dB})-L_{\text {pathloss }}(\mathrm{dB})$ (5) 
The underwater signal propagation depends on the path loss in water. Friis equation $[12,8]$ gives the received power as a function of transmitted signal, path loss and antenna gain at the receiver as shown above in (5) where $P_{t}$ is the transmit power, $G_{r}$ and $G_{t}$ are the gains of the receiver and transmitter antenna, $L_{\text {pathloss }}$ is the path loss in water [7]. The path loss is shown in (6).

$L_{\text {pathloss }}(\mathrm{dB})=L_{o}(\mathrm{~dB})+L_{w}(\mathrm{~dB})+L_{\text {att }}(\mathrm{dB})$

where $L_{o}$ is the path loss in air and given by

$L_{o}(\mathrm{~dB})=20 \log \left(\frac{4 \pi d f}{c}\right)$

Where $d$ is the distance between transmitter and receiver in meters, $\mathrm{f}$ is the operating frequency in Hertz and $c$ is the velocity of light in air in meters per second.

$L_{w}(\mathrm{~dB})$ is the path loss due to change in medium and is given by $[9,14]$ :

$L_{w}(\mathrm{~dB})=20 \log \left(\frac{\lambda_{o}}{\lambda}\right)$

where $\lambda_{0}$ is the signal wavelength in air and calculated as $\lambda_{o}=c / f$ and $\lambda$ is the wave factor given by $\lambda_{o}=2 \lambda / \beta$ and $\beta$ is the phase shifting constant and calculated as

$\beta=\omega \sqrt{\frac{\mu \varepsilon}{2}\left(\sqrt{1+\left(\frac{\dot{\varepsilon}}{\varepsilon}\right)^{2}}\right)+1}$

Where $\varepsilon$ and $\varepsilon$ are the real and imaginary parts of the complex dielectric constant. $L_{a t t}(\mathrm{~dB})$ is the path loss due to attenuation in medium and given by

$L_{a t t}(\mathrm{~dB})=10 \log \left(\mathrm{e}^{-2 \alpha d}\right)$

where $\alpha$ is the attenuation constant and calculated as

$\alpha=\omega \sqrt{\frac{\mu \varepsilon}{2}\left(\sqrt{1+\left(\frac{\dot{\varepsilon}}{\varepsilon}\right)^{2}}\right)-1}$

According to (5), the received sensing power of a sensor at distance $r$ from a target can be expressed as in [12]

$P_{\text {rec }}(\mathrm{dBm})=$

$P_{t}(\mathrm{dBm})+G_{t}(\mathrm{~dB})+G_{r}(\mathrm{~dB})-L_{\text {pathloss }}(\mathrm{dB})+x_{\sigma}$

where $x_{\sigma}$ is a Gaussian random variable with zero mean and variance $\sigma^{2}$ and represents shadowing effects in the propagation path.

The probability that a sensor $S$ detects an event occurring at $r$ distance can be expressed as

$P_{d}(r)=\left(P_{r e c}>\lambda\right)$

where $\lambda$ is the power threshold value (where $P_{\text {rec }}$ has been defined in (12).

$P_{d}(r)=P\left(x_{\sigma}>\lambda-P_{t}(\mathrm{dBm})-G_{t}(\mathrm{~dB})-G_{r}(\mathrm{~dB})+\right.$

$\left.L_{\text {pathloss }}(\mathrm{dB})\right)$

where $P($.$) denotes probability function. Therefore, the$ detection probability $P_{d}(r)$ can be expressed as

$P_{d}(r)=\int_{\lambda-b}^{\infty} \frac{1}{\sqrt{2 \pi \sigma^{2}}} \mathrm{e}^{\left[-\left(x^{2} / 2 \sigma^{2}\right)\right]} d x$

where $\mathrm{b}=P_{t}(\mathrm{dBm})+G_{t}(\mathrm{~dB})+G_{r}(\mathrm{~dB})-L_{\text {pat hloss }}(\mathrm{dB})$

From (15)

$P_{d}(r)=Q\left(\frac{\lambda-b}{\sigma}\right)$
$P_{d}(r)=Q\left(\frac{\lambda-P_{t}-G_{t}-G_{r}+L_{\text {pat }} \text { hloss }}{\sigma}\right)$

(16)Sensor $S$ to be located at a distance $r$ from the target located at the origin of the sphere. $R_{\max }$ is taken as the maximum sensing range of a sensor and $R$ is the average sensing radius of a sensor. $r$ is assumed to be continuous and $d r$ approaches 0 . The probability that the target is detected by an arbitrary sensor placed in the specified volume $4 \pi r^{2} d r$ of network volume V (refer to Figure 1) can be calculated as follows:

$P_{d}=\frac{1}{V} \int_{r=0}^{R_{\max }} P_{d}(r) \times 4 \pi r^{2} d r$

Now, the coverage probability can be calculated as follows. By substituting $P_{d}(r)$ into (17), the probability that a target is sensed by an arbitrary sensor node placed in the specified volume of surface area $4 \pi r^{2} d r$ of network volume $\mathrm{V}$ can be expressed as

$P_{d}=\frac{1}{V} \int_{r=0}^{R_{\max }} Q\left(\frac{\lambda-P_{t}-G_{t}-G_{r}+L_{p a t} \text { hloss }}{\sigma}\right) \times 4 \pi r^{2} d r$

According to (1) and (2) the coverage probability $P_{c}$ can be expressed as

$P_{c}=1-\exp \left(-\frac{N}{V} \int_{0}^{R_{\max }} 4 \pi r^{2} Q\left(\frac{\lambda-P_{t}-G_{t}-G_{r}+L_{\text {pat hloss }}}{\sigma}\right) d r\right)$

This equation presents the coverage probability for a specific number of sensors to be deployed.

\section{RESULTS AND ANALYSIS}

The simulation and numerical results showing the impact of sensing channel model under shadowing environment on the network coverage are presented. In the simulation, the sensing field is assumed to be a cube with volume $\mathrm{V}=50 \times 50 \times 50$ $\mathrm{m}^{3}$.The maximum sensing radius $R_{\max }$ is taken as $10 \mathrm{~m}$. The parameters along with their values as used in simulation are shown in Table 1.

Figure 2 illustrates the results of detection probability $P_{d}$ versus the sensing radius $r$ for different frequencies in shadowing environment for electromagnetic signal. These results are obtained by applying the derivation given in equation (16). The graph shows the relationship between frequency, sensing radius and detection probability. From this graph we find that attenuation is proportional to frequency of the signal. Therefore, as we increase the frequency of the signal, the sensing radius keeps decreasing but detection probability is enhanced. Sensing radius refers to the distance the sensors can keep track of. The results shown in the above figures have been obtained by keeping shadowing constant. This shows that in case of shadowing environment, higher is the frequency lower is the sensing radius of the sensors. The detection probability increases with an increase in frequency in case of constant shadowed environment. This indicates that in the shadowing environment, detection probability enhances with increasing frequency but with a downgrade in the sensing radius. 


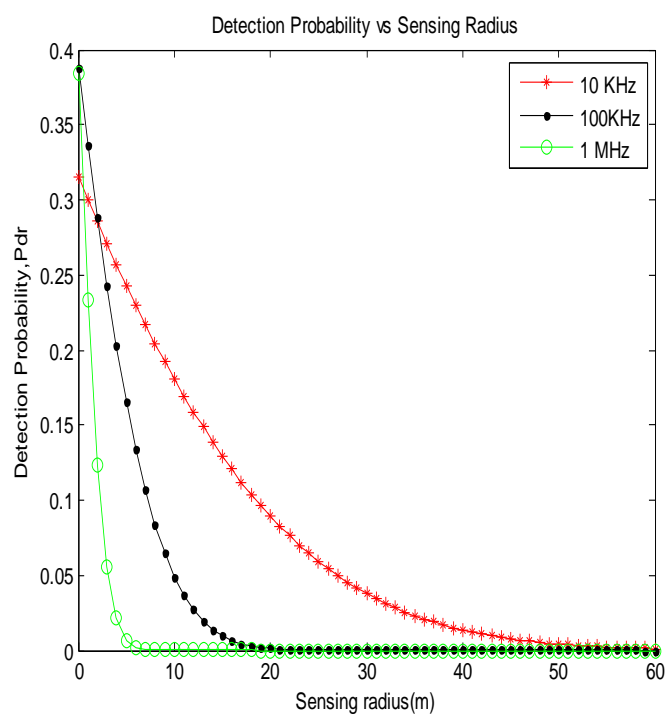

Figure 2. Detection probability vs sensing radius

Figure 3 depicts the relationship between sensing radius and detection probability for different values of shadowing parameter with constant frequency of the signal. As in this work shadowing follows log normal distribution, therefore with the increase in the shadowing parameter, the probability for shadowing to occur decreases (i.e. shadowing decreases). As shadowing decreases the sensing radius $R$ keeps increasing and detection probability increases. It can be inferred from the below figure that the detection probability increases and sensing radius also increases as shadowing parameter decreases underwater. With the degradation of shadowing effects the detection probability increases. Therefore in underwater environment as shadowing decreases detection probability becomes higher.

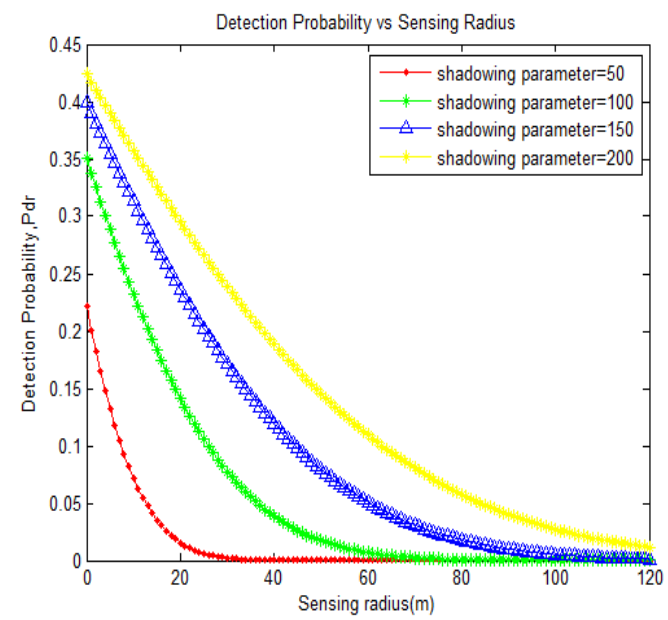

Figure 3: Detection Probability vs. Sensing Radius

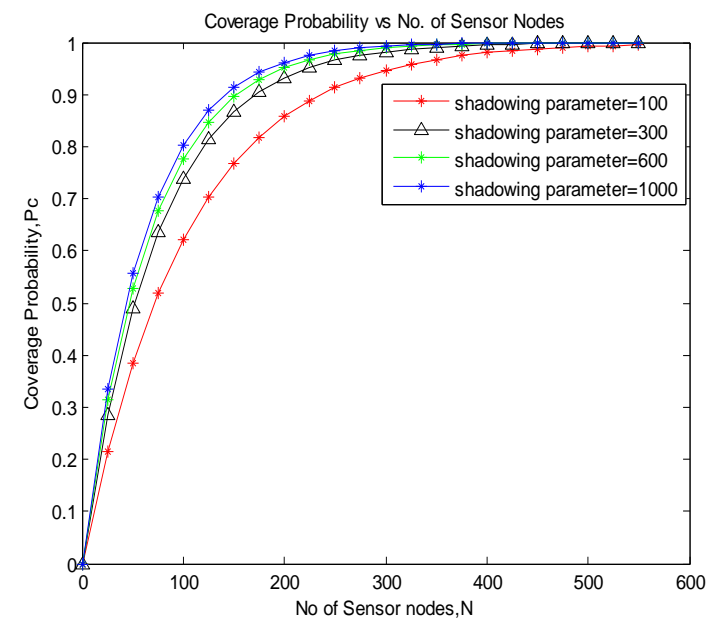

Figure 4a: Coverage Probability vs no of sensor nodes

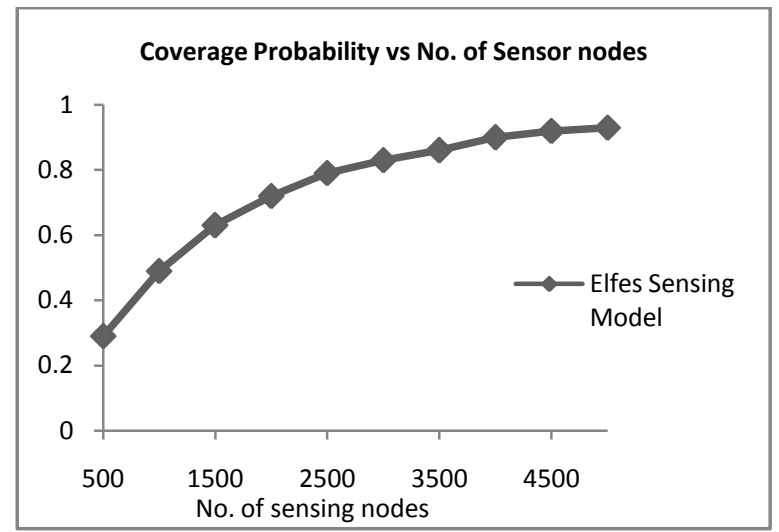

Figure 4 b: Elfes Sensing Model

The results in figure 4a show the impact of number of sensor nodes on the coverage probability for different shadowing parameters for EM signals. From the above figures, we observe that as the value of shadowing parameter increases (i.e. shadowing decreases), the number of sensor nodes required for the full coverage (i.e. $=1$ ) decreases. With the degradation of shadowing, the coverage probability goes up and the number of nodes needed to achieve the same also decreases. As sigma ( $\sigma$, shadowing parameter) increases, shadowing decreases, and therefore number of nodes to provide full coverage decreases. A comparison with the well known Elfes sensing model has been given which needs about 4500 nodes to achieve a coverage probability of 1 . Elfes model (figure 4b) deals with terrestrial sensor networks whereas we are dealing with underwater sensor networks. As little work has been done in the area of underwater sensor networks channel model, the comparison has been done with Terrestrial sensor networks model. As can be seen in the figure, the number of sensor nodes is extremely high (about 4500) in case of Elfes model to achieve a coverage probability of one but in our model despite taking the impact of shadowing into consideration the number of sensors needed is very less (around 500 for highest shadowing). 
Table 1. Parameters Taken

\begin{tabular}{|c|c|}
\hline Parameters & Values \\
\hline $\begin{array}{l}\text { No. of } \\
\text { sensors }\end{array}$ & 0 to 550 \\
\hline $\begin{array}{l}\text { C (speed of } \\
\text { light) }\end{array}$ & $3 \times 10^{8} \mathrm{~m} / \mathrm{s}$ \\
\hline $\begin{array}{l}\text { Shadowing } \\
\text { parameter }\end{array}$ & 200 \\
\hline $\begin{array}{l}\mathrm{G}_{\mathrm{t}} \text { (transmitter } \\
\text { antenna gain) }\end{array}$ & $3 \mathrm{~dB}$ \\
\hline $\begin{array}{l}\mathrm{G}_{\mathrm{r}} \text { (transmitter } \\
\text { receiver gain) }\end{array}$ & $3 \mathrm{~dB}$ \\
\hline $\begin{array}{c}\text { Transmitted } \\
\text { power } \mathrm{P}_{\mathrm{t}}\end{array}$ & $126 \mathrm{~dB}$ \\
\hline $\begin{array}{l}\text { Threshold } \\
\text { power }\end{array}$ & $16 \mathrm{~dB}$ \\
\hline $\begin{array}{c}\mu(\text { magnetic } \\
\text { permeability) }\end{array}$ & $\begin{array}{c}0.0000012 \\
56627\end{array}$ \\
\hline $\begin{array}{c}\varepsilon \text { (dielectric } \\
\text { constant) }\end{array}$ & $\begin{array}{c}0.0000000 \\
00708\end{array}$ \\
\hline
\end{tabular}

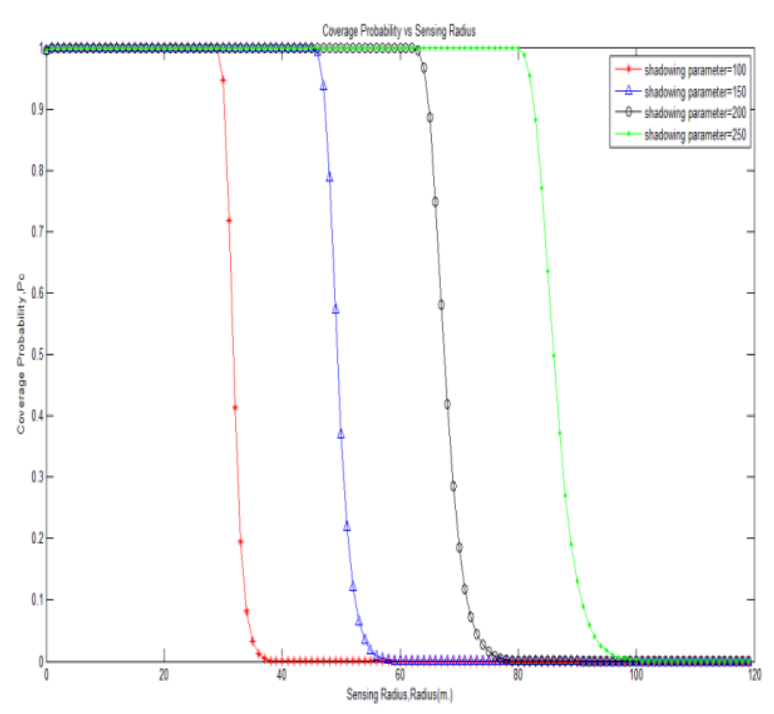

Figure 5: Coverage probability vs Sensing Radius for varying shadowing parameters

Figure 5 shows the impact of sensing radius on the coverage probability for different value of shadowing parameter. From the results, we observe that as shadowing parameter increases, (i.e. as shadowing decreases) the sensing radius increases and coverage probability remains one for larger sensing radius. Therefore we can infer that less is the shadowing greater is the area that can be sensed underwater and higher is the coverage probability of the sensor network.

\section{CONCLUSION}

In this paper, a new sensing channel model considering shadowing fading for coverage of underwater sensor networks has been suggested. A model for determining detection probability and coverage probability in underwater sensor networks has been modelled. It is noted from the results that decrease in shadowing results in greater probability of coverage as greater is the radius that can be sensed. It is also observed that the required number of sensor nodes increases for desired coverage where fading effects are more pronounced. Also a coverage probability of one can be obtained with much less number of sensor nodes than the terrestrial model. It is observed that full coverage of the sensor networks can be achieved with around 500 sensors even in high shadowing environment. It is evident that the proposed sensing model provides good network coverage for a real environment as compared to other probabilistic sensing models existing for terrestrial sensor networks. Directions for future work include generalization of the results to more distributions and a study of the impact of various other parameters on the results found. As little work has been done in the area of underwater sensor networks, the proposed model can be investigated in the future to evaluate the performance of underwater wireless sensor networks in realistic environment. Further, this model could also be used to investigate sensing coverage considering other interference effects.

\section{REFERENCES}

[1] A.Ghosh, S.Das "Coverage and connectivity issues in wireless sensor networks: A survey". Pervasive and Mobile Computing Elsevier.2008.

[2] G.Mao, B.Anderson, B.Fidan "Path loss exponent estimation for wireless sensor network localization" Computer Networks.2007.

[3] C.Zhu, C.Zheng, L.Shu, G.Han"A survey on coverage and connectivity issues in wireless sensor networks" Journal of Network and Computer Applications, 2012.

[4] Xing G, Wang X, Zhang Y, Lu C, Pless R, Gill C. Integrated coverage and connectivity configuration in wireless sensor networks. In Proceedings of the 1st International Conference on Embedded Networked Sensor Systems (SenSys '03);2003

[5] G.Xing, X.Wang, Y.Zhang, C.Lu, R.Pless, C.Gill "Integrated coverage and connectivity configuration for energy conservation in sensor networks". ACM Transactions on Sensor Networks. 2005.

[6] Y.Tsai "Sensing coverage for randomly distributed wireless sensor networks in shadowed environments" IEEE Transactions on Vehicular Technology. 2008.

[7] L.Liu, S.Zhou, J.Cui "Prospects and Problems of Wireless Communication for Underwater Sensor Networks" Wireless Communication \& Mobile Computing. 2008.

[8] Elrashidi A. Underwater Wireless Sensor Network Communication Using Electromagnetic Waves at Resonance Frequency 2.4 GHz. In Proceedings of the 15th Communications and Networking Simulation Symposium

[9] T. Weldon, A. Rathroe "Wave Propagation Model and Simulation for Landmine Detection" Tech. Republic 1999.

[10] Stuber. G. Principles of Mobile Communication. Klumer Academic Publishers.1996.

[11] Theodore, Rappaport S. Wireless Communications: Principles \& Practice, New Jersey :Prentice Hall,1996.

[12] Ramo S, Whinnery J, Van Duzer T. Fields and Water for Communications Electronics. New York : John Wiley and Sons.1994. 
[13] Wait J. Electromagnetic Wave Theory. New York :Harper and Row. 1985.

[14] Y.Xiaoqing "Electromagnetic Wave Propagation In Soil For Wireless Underground Sensor Networks". Progress In Electromagnetics Research 2013.

[15] K.Hunt, J.Niemeier, A.Kruger "RF Communications in Underwater Wireless Sensor Networks" IEEE International Conference on Electro/Information Technology (EIT) 2010

[16] J.Goh,A.Shaw,A.Shanmma "Underwater Wireless Communication System”. Journal of Physics.2009

[17] A.Shamma, A.Shaw,S.Saman "Propagation of Electromagnetic Waves at $\mathrm{MHz}$ Frequencies through Seawater". IEEE Transactions on Antennas and Propagation; 2004

[18] Heidemann J, Stojanovic M, Zorzi M Underwater sensor networks: applications, advances and challenges [Internet] 2011[cited

http://rsta.royalsocietypublishing.org/content/370/1958/1 58

[19] Hossain A, Biswas P, Chakrabarti S.Sensing models and its impact on network coverage in wireless sensor network. In Proceedings of the IEEE Region 10 Colloquium and $3^{\text {rd }}$ International Conference on Industrial and Information Systems(ICIIS '08)2008 December; Kharagpur, India ;P 1-5.

[20] C.Huang,Y.Tseng "The coverage problem in a wireless sensor network". Mobile Networks and Applications.2005

[21] S.Kumar, D.K. Lobiyal "Sensing Coverage Prediction for Wireless Sensor Networks in Shadowed and Multipath Environment". The Scientific World Journal.2013. 